\title{
Caracterização da Comunicação entre Progenitores e Filhos em Idade Escolar: Estudo com uma Amostra Portuguesa ${ }^{1}$
}

\author{
Alda Marques Portugal ${ }^{2}$ \\ Isabel Marques Alberto \\ Universidade de Coimbra
}

\begin{abstract}
RESUMO - A comunicação parento-filial é uma dimensão central do exercício da parentalidade. O objetivo do presente estudo foi identificar e comparar as percepções de 467 progenitores e 329 crianças (entre 7 e 11 anos) sobre a comunicação parentofilial, através da Escala de Avaliação da Comunicação na Parentalidade (COMPA). Os testes $t$ de Student, ANOVA e correlação de Pearson revelaram alguns resultados estatisticamente significativos: destaque positivo de mães/filhas na comunicação, relação positiva entre dimensões metacomunicação e partilha/confiança da intimidade por parte das crianças, e influência das variáveis sociodemográficas (níveis socioeconómico e de escolaridade baixos, contexto rural) sobre a comunicação. De uma forma geral, os resultados sugerem a necessidade de dar atenção às relações parento-filiais em contextos escolares e socioeconómicos desfavorecidos.
\end{abstract}

Palavras-chave: comunicação pais-criança, crianças em idade escolar, estudo comparativo

\section{Characterization of Parent-Child Communication in School-age Children: Study with a Portuguese Sample}

\begin{abstract}
Parent-child communication is a central dimension of the parenting task. The main goal of the present study was to identify and compare the perceptions of 467 parents and 329 children (ages between 7-11 years) related to parent-child communication, using the Perception Scale of Parenting Communication. Student's t-test, ANOVA, and Pearson correlations revealed some statistically significant results: a positive emphasis of mothers/daughters on communication, a positive relationship between meta-communication and sharing/confidence dimensions for children, and the influence of sociodemographic variables (low levels of financial income and education, rural context) on the quality of communication. In general, these results seem to indicate the necessity of paying attention to the parent-child relationship in disadvantaged school and socioeconomic contexts.
\end{abstract}

Keywords: parent-child communication, school-age children, comparative study

A relação parento-filial inicia-se a partir do momento da concepção de um filho, acontecimento que marca o estabelecimento da primeira ligação intergeracional do ser humano (Segrin \& Flora, 2005). Esta relação é constituída e definida por diversas dimensões das quais se destaca a comunicação (Carr, 2006). Por comunicação entende-se o processo contínuo de transmissão de informação que integra diferentes realidades e sociedades/culturas (Fiske, 1993/2005) sendo que, no contexto particular da família, a comunicação permite reconhecer e dar resposta às necessidades/exigências básicas da parentalidade em função de cada etapa do ciclo vital (Carr, 2006; Relvas, 1996). A comunicação familiar engloba diversas dimensões: expressão do afeto e do apoio emocional, que diz respeito à troca de mensagens positivas entre os membros da família, à clareza comunicacional, à resolução de problemas, ao suporte emocional, ao apoio verbal e à demonstração de afeto e empatia (Ségrin \& Flora, 2005); disponibilidade, que se refere à abertura comunicacional e à sinceridade no diálogo (Kirkman, Rosenthal, \& Feldman,

1 Apoio: Fundação para a Ciência e a Tecnologia, Ministério da Educação, Portugal.

2 Endereço para correspondência: Urbanização Rio Novo, lote 248, Nazaré, Portugal, CEP 248, 2450-076.

E-mail: aaldaportugal@gmail.com
2005); metacomunicação, que está relacionada com o esclarecimento dos conteúdos comunicacionais verbalizados (Watzlawick, Beavin, \& Jackson, 1967/1993); e confiança/ partilha equilibrada de questões e problemas pessoais sobre trabalho, relacionamentos, amizades e família (Kirkman et al., 2005). A etapa do ciclo vital "família com filhos em idade escolar" é considerada uma das mais desafiantes em termos do exercício da parentalidade (Relvas, 1996). Empiricamente, registam-se alguns estudos centrados no relacionamento pais-filhos durante essa etapa, por exemplo ao nível das práticas e dos estilos parentais (e.g., Camacho \& Matos, 2007; Kerr, Stattin, \& Özdemir, 2012) no entanto, poucos são aqueles que analisam a dimensão comunicacional de forma específica (Stamp, 2004).

Numa perspectiva sistémica, a entrada das crianças na escola é "um momento capital de abertura do sistema familiar ao mundo extrafamiliar. (...) É a primeira crise de desmembramento com que a família se confronta" (Relvas, 1996, p. 114). Essa etapa do ciclo vital, que contempla os primeiros seis anos de escolaridade (Stafford, 2004), implica a realização de tarefas específicas tanto por parte dos pais (garantir a segurança da criança; prestar-lhe os cuidados básicos, incluindo a dimensão afetiva e relacional; monitorizar o seu comportamento; estimulá-la intelectualmente), como por parte dos filhos (obter sucesso escolar; iniciar o 
processo de socialização com o grupo de pares) (Carr, 2006). De acordo com Relvas (1996), essa fase da vida familiar conjuga movimentos centrípetos (fecho ao exterior) com movimentos centrífugos (abertura ao exterior), dando início a uma das tarefas mais relevantes: o processo de socialização e individuação dos filhos (Herbert, 2004; Taborda Simões, Martins, \& Formosinho, 2006). Em termos comunicacionais, verifica-se uma continuidade projetiva das regras de comunicação intrafamiliares para outros subsistemas (e.g., pares, escola), passando a criança a assumir o papel de gestor comunicacional (Alarcão 2006; Relvas, 1996). Nessa gestão, destacam-se três áreas importantes (Stafford, 2004): a) os comportamentos afetivos (suporte emocional e conforto), que refletem a capacidade da criança para perceber e dar apoio perante a expressão de sentimentos negativos como tristeza, medo, ansiedade e raiva; b) as competências de argumentação e de gestão de conflitos necessárias para superar e resolver situações relacionais entre pares; e c) a expressão afetiva/emocional, que diz respeito à competência das crianças para gerirem a manifestação de emoções com os pares (automonitorização).

Streeck (2002) refere que a comunicação familiar é um dos agentes instrumentais mais importantes para o processo de socialização infantil, fato que é reforçado pelo estudo de Gentzler, Contreras-Grau, Kerns e Weimer (2005). Esses autores verificaram que a competência social das crianças em idade escolar tende a ser maior em função do uso de estratégias de coping adaptativas, sendo que o recurso a essas estratégias depende da comunicação aberta mantida com os progenitores no contexto familiar. Riesch, Anderson e Krueger (2006) constataram que o processo de comunicação parento-filial, que inclui a abertura comunicacional, a satisfação com o sistema familiar, o cuidar do outro e a capacidade de resolução de conflitos, pode funcionar como mecanismo mediador e protetor de comportamentos de risco das crianças em idade escolar, tais como, consumo de tabaco, álcool, drogas leves ${ }^{3}$ e iniciação sexual precoce. Assim, Riesch et al. (2006) defendem que a intervenção na comunicação entre progenitores e filhos pode prevenir o desenvolvimento de comportamentos de risco ao nível da saúde (e.g., abuso de substâncias). Hillaker, Brophy-Herb, Villarruel e Haas (2008) desenvolveram uma investigação com o objetivo de analisar o efeito da comunicação familiar sobre o desenvolvimento de competências sociais e constataram que, de fato, a manutenção de uma comunicação parento-filial positiva (e.g., responsividade comunicacional) contribui para o desenvolvimento de competências sociais, tais como: valores sociais positivos (cuidar do outro, sentido de igualdade e justiça, integridade, honestidade, responsabilidade), tomada de decisão, competência interpessoal e competências de resiliência. Bumpus e Hill (2008), através de um estudo longitudinal, analisaram a relação entre a existência de segredos por parte dos filhos e a comunicação que estes mantêm com os seus progenitores, com o ajustamento social das crianças. Os

3 Os autores definem drogas leves da seguinte forma “ (...) drug use generally refers to accepting street drugs, smoking marijuana, and inhaling fumes of butane, glue, or gasoline to get high" (Riesch, Anderson e Krueger, 2006, p.42) resultados indicam que existe uma relação moderada entre a existência do segredo sobre as atividades diárias por parte das crianças e a frequência da comunicação parento-filial. Verifica-se uma menor comunicação entre progenitores e filhos quando estes ocultam informação aos seus pais. Além disso, constatou-se, que as crianças que guardam segredos em relação às figuras parentais tendem a ser percecionadas pelos professores como sendo menos competentes a nível social, mais opositoras e agressivas.

A literatura demonstra que a comunicação entre progenitores e filhos é influenciada por diversas variáveis, tais como: sexo, estrutura familiar, nível socioeconómico, cultura/local de residência e nível de escolaridade (Segrin \& Flora, 2005). Relativamente às diferenças em relação ao sexo, McNaught (2000) concluiu que: os progenitores de ambos os sexos tendem a comunicar mais abertamente com as filhas do que com os filhos, e as filhas tendem a ser mais expressivas (quando se referem a sentimentos) do que os filhos, já que estes manifestam maior contenção na expressão emocional. Também Patrick, Snyder, Schrepferman e Snyder (2005) verificaram que as meninas reportam uma comunicação mais frequente com ambos os progenitores do que os meninos. Ao nível da estrutura familiar, Dunn, Davies, O'Connor e Sturgess (2001) constataram que as crianças de famílias reconstituídas desenvolvem relações de menor confiança com ambos os progenitores, comparativamente às crianças de famílias nucleares intactas, enquanto as crianças de famílias monoparentais tendem a partilhar mais atividades com a família do que crianças que vivem noutras composições familiares. Alguns autores verificaram que a comunicação parento-filial é, também, negativamente afetada pela ocorrência de um divórcio (Afifi, Huber, \& Ohs, 2006; Eldar-Avidan, Haj-Yahia, \& Greenbaum, 2009; McManus \& Nussbaum, 2011; Nair \& Murray, 2005). O nível socioeconómico influencia, também, o exercício da parentalidade (Magnuson \& Duncan, 2002). De acordo com Magnuson e Duncan (2002), os progenitores com baixos rendimentos econômicos tendem a recorrer a estratégias educativas mais centradas no castigo físico e em estilos comunicacionais mais autoritários que não contemplam a perspectiva das crianças. Da mesma forma, a comunicação parece ser mediada pelo contexto social em que a família vive. As crenças e as atitudes comunicacionais distinguem-se e adaptam-se em função do contexto geográfico seja ele rural, urbano ou semiurbano. O estudo de Wamoyi, Fenwick, Urassa, Zaba e Stones (2010) evidenciou que, apesar dos filhos das famílias que viviam em contexto rural conversarem com os progenitores sobre aspectos relacionados com a sexualidade, reportavam falta de confiança sobre o que partilhar e com qual dos progenitores partilhar, por receio de serem castigados. Os progenitores, por sua vez, referem dificuldade na comunicação sobre esse tópico devido às barreiras culturais e à falta de conhecimento. Jiménez e Delgado (2002) verificaram que também o nível de literacia parental tem implicações na comunicação parento-filial, constatando que quanto menor for a escolaridade dos progenitores menos frequentes serão as interações comunicacionais.

Atendendo à importância que a comunicação tem no contexto do exercício da parentalidade, o principal objetivo do presente estudo é identificar e comparar as percepções que progenitores e crianças (entre os 7 e os 11 anos) têm sobre 
a comunicação parento-filial, através de um instrumento de medida específico da comunicação: Escala de Avaliação da Comunicação na Parentalidade (COMPA). De acordo com esse objetivo, foram colocadas três hipóteses de investigação: 1) existem diferenças de gênero, no sentido dos elementos do sexo feminino (mães e filhas) manterem uma comunicação parento-filial mais frequente do que os elementos do sexo masculino (pais, filhos) (Bumpus \& Hill, 2008; McNaught, 2000; Patrick et al., 2005); 2) existe uma relação positiva entre uma comunicação parento-filial clara (metacomunicação) e a confiança/partilha de situações problemáticas por parte dos filhos em relação aos progenitores (Bumpus \& Hill, 2008; Patrick et al., 2005); e 3) variáveis sociodemográficas como a "estrutura familiar" (Afifi et al., 2006; Dunn et al., 2001; Eldar-Avidan et al., 2009; McManus \& Nussbaum, 2011; Nair \& Murray, 2005), o "nível socioeconómico" (Magnuson \& Duncan, 2002), o "local de residência" (Wamoyi et al., 2010) e a "escolaridade" (Jiménez \& Delgado, 2002) dos participantes influenciam os padrões comunicacionais entre progenitores e filhos.

\section{Método}

\section{Amostra}

A amostra total é constituída por 796 participantes distribuídos por duas subamostras independentes: 329 crianças com idades entre 7 e 11 anos $(M=9,68)$ e 467 progenitores com idades entre 24 e 67 anos $(M=39,41)$.

A subamostra das crianças é composta por 145 (44,1\%) meninos e $184(55,9 \%)$ meninas, verificando-se que 32 crianças $(9,7 \%)$ vivem num contexto predominantemente urbano, $170(51,7 \%)$ num contexto medianamente urbano e 127 $(38,6 \%)$ num contexto predominantemente rural. Ao nível do estatuto socioeconómico, calculado com base nos indicadores disponíveis no Instituto Nacional de Estatística relativos às profissões e rendimentos dos progenitores, constata-se que $18(5,5 \%)$ crianças pertencem à classe socioeconómica baixa, $281(85,4 \%)$ à classe média e $29(8,8 \%)$ à classe alta. Por fim, relativamente à escolaridade, $174(52,9 \%)$ crianças frequentam os quatro anos iniciais de escolaridade, 154 (46,8\%) estão a terminar os seis anos de escolaridade e apenas uma $(0,3 \%)$ das crianças está a completar os nove anos de escolaridade. Em termos da estrutura familiar, verifica-se que 278 (84,5\%) crianças vivem em agregados familiares nucleares intactos, $29(8,8 \%)$ em famílias pós-divórcio, 18 (5,5\%) crianças vivem em famílias reconstituídas e quatro $(1,2 \%)$ crianças vivem em famílias com apenas um progenitor (monoparentais). Em média, os participantes dessa subamostra têm um irmão $(M=1,39, D P=0,84)$.

A subamostra dos progenitores é composta por 84 pais (18,0\%) e 383 mães (82,0\%), verificando-se que 225 (48,2\%) progenitores vivem num contexto predominantemente urbano, $184(39,4 \%)$ num contexto medianamente urbano e 50 (10,7\%) habitam num contexto predominantemente rural. Relativamente ao nível socioeconômico, 345 participantes (73,9\%) enquadram-se no nível socioeconômico médio, 89 $(19,1 \%)$ pertencem ao nível socioeconômico baixo e 28 (6\%) estão no nível socioeconômico alto. Considerando a escolaridade verifica-se que $21(4,5 \%)$ progenitores completaram quatro anos de escolaridade, $25(5,4 \%)$ completaram seis anos, $49(10,5 \%)$ dos progenitores concluíram nove anos e $118(25.3 \%)$ terminaram 12 anos (escolaridade obrigatória em Portugal). Grande parte dos progenitores que compõem esta amostra $(n=175,37,5 \%)$ têm uma licenciatura e 58 têm uma pós-graduação (mestrado: $n=37,7,9 \%$; doutoramento: $n=21,4,5 \%$ ). Constata-se que a maioria dos progenitores representa famílias nucleares intactas $(n=403,86,3 \%), 48$ $(10,3 \%)$ participantes vivem em famílias pós-divórcio, nove $(1,9 \%)$ estão numa família reconstituída e apenas sete $(1,5 \%)$ progenitores pertencem a um núcleo familiar monoparental. Os progenitores desta amostra têm, em média, dois filhos ( $M$ $=1.92, D P=0.73$ ) e pelo menos um deles encontra-se no intervalo de idades entre 7 e 11 anos.

\section{Instrumentos}

\section{Ficha de dados sociodemográficos}

Trata-se de um questionário simples e breve que inclui questões de recolha de informação relacionadas com o sexo (masculino/feminino), idade (em anos), papel familiar (pai/ mãe/filho/filha), número filhos/irmãos, local de residência (distrito), nível socioeconómico (alto/médio/baixo) e tipo de constituição familiar (nuclear/pós-divórcio/monoparental/ reconstituída).

\section{Escala de Avaliação da Comunicação na Parentalidade}

É um instrumento de autorrelato que avalia os padrões de comunicação entre progenitores e filhos através de uma escala de tipo Likert ( $1=$ Nunca; $5=$ Sempre $)$. A uma pontuação mais elevada corresponde uma percepção mais positiva da comunicação estabelecida entre progenitores e filhos. Neste estudo usou-se a versão parental (COMPA-P), e a versão para filhos dos 7-11 anos (COMPA-C) (Portugal \& Alberto, no prelo)

A versão parental da escala COMPA é composta por 44 itens distribuídos por cinco subescalas (expressão do afeto e apoio emocional, disponibilidade para a comunicação, metacomunicação, confiança/partilha comunicacional dos pais para os filhos, confiança/partilha comunicacional dos filhos para os pais). Essa versão obteve um valor de consistência interna de $\alpha=0,91$. A versão COMPA-C tem 16 itens distribuídos por duas subescalas (disponibilidade dos pais para comunicar com os filhos, expressão do afeto e apoio emocional) e registou um valor de $\alpha=0,88$ nos estudos relativos à consistência interna. A versão para as crianças é respondida de forma separada para cada um dos progenitores.

\section{Procedimento}

Depois da aprovação do estudo por parte da Direção de Serviços de Inovação Educativa (departamento do governo português), este foi divulgado, via e-mail, por 15 escolas públicas distribuídas pelo país, das quais sete aceitaram 
participar. Essas escolas de ensino básico correspondem a um universo de cerca de 720 alunos com idades entre os 7 e os 11 anos de idade e estão distribuídas por três municípios localizados na zona centro de Portugal: Coimbra, Leiria e Alcobaça. Nas escolas que aceitaram participar, distribuiu-se pelos alunos um documento informativo sobre o estudo, os seus objetivos e o consentimento informado a assinar pelos respectivos progenitores. O protocolo de avaliação, aplicado por um elemento da equipa de investigação ou por um docente, foi respondido coletivamente em contexto de sala de aula. No caso das crianças mais pequenas ( 7 e 8 anos) os itens do questionário foram lidos, um a um, em voz alta para todos os alunos e a escala de Likert foi inicialmente explicada com recurso a exemplos práticos. Por sua vez, a aplicação do protocolo aos progenitores foi feita de duas formas: em formato de papel e lápis ( $n=290$, amostra de conveniência) e numa versão online ( $n=177$, criação de um website). Na primeira situação (formato de papel e lápis) o protocolo foi aplicado presencialmente e o preenchimento foi efetuado em casa dos participantes ou no seu local de trabalho, em função do que lhes era mais conveniente. Foi solicitado a esses progenitores que pensassem na comunicação que estabelecem com um dos seus filhos, em particular aquele que se encontra na faixa etária dos 7 aos 11 anos. Nas situações em que os progenitores tinham mais do que um filho nessa faixa etária, pediu-se para que respondessem especificamente sobre um dos filhos, explicando-se que os padrões comunicacionais diferem de filho para filho. O processo de recolha dessa subamostra foi baseado no método não-probabilístico de "bola de neve", ou seja, foi gerada uma rede de contatos a partir da aplicação do protocolo aos primeiros progenitores (Pais-Ribeiro, 2007). Importa referir que em alguns casos se registam missings nas respostas à escala, aspecto que provoca ligeiras variações nos valores de $n$ quando das comparações entre grupos.

As amostras dos progenitores e das crianças são independentes, não havendo relação de parentesco entre si. Essa opção deveu-se a dois motivos: à maior acessibilidade a participantes independentes e à possibilidade de controlo da manipulação das respostas, condição difícil de gerir caso os questionários fossem aplicados a todos os elementos do mesmo agregado familiar (comparação de respostas).

\section{Resultados}

\section{Hipótese 1: Existem diferenças ao nível da variável "sexo": mães e filhas mantêm uma comunicação parento-filial mais frequente do que os pais e filhos}

Para analisar a influência da variável sexo de progenitores e filhos na comunicação parento-filial recorreu-se ao teste de comparação de médias $t$ de Student para amostras independentes.

\section{Resultados na amostra das crianças}

Os resultados revelaram que as subescalas da versão filial da escala COMPA têm variâncias homogéneas, registando-se diferenças estatisticamente significativas na subescala "disponibilidade dos pais para comunicar com os filhos", quer tendo por referência o pai $[t(309)=-2,648$, $p=0,009]$, quer a mãe $[t(313)=-2,331, p=0,020]$. Em média, as crianças do sexo feminino percebem uma maior disponibilidade parental à comunicação por parte da mãe $(M$ $=37,15, D P=3,64)$ e do pai $(M=35,96, D P=4,19)$, comparativamente às crianças do sexo masculino $[(M=36,14$, $D P=4,00),(M=34,69, D P=4,20)]$. Porém, a magnitude das diferenças entre as médias nos filhos revelou-se pequena, quer em relação à mãe $\left(\eta^{2}=0,021\right)$, quer em relação ao pai $\left(\eta^{2}=0,016\right)$. Esses resultados podem ser analisados em detalhe na Tabela 1 .

\section{Resultados na amostra dos progenitores}

O teste $t$ de Student para amostras independentes foi utilizado para comparar as respostas dos progenitores. Verificada a homogeneidade das variâncias constatou-se, tal como se pode ver na Tabela 1, a existência de três valores estatisticamente significativos, nomeadamente ao nível do resultado global da escala $[t(356)=-2,057, p=0,040]$, da subescala "expressão do afeto e apoio emocional" $[t(450)$ $=-3,182, p=0,002]$ e da subescala "confiança/partilha comunicacional de filhos para pais" $[t(454)=-3,505, p=$ $0,001]$. Em média, as mães $(M=184,26, D P=14,11)$ têm uma percepção mais positiva da comunicação do que os pais $(M=180,26, D P=15,14)$, destacando-se particularmente nas subescalas "expressão do afeto e apoio emocional" $(M$ $=53,69, D P=4,12)$ e "confiança/partilha comunicacional de filhos para os pais" ( $M=28,23, D P=3,20)$ quando comparadas com os pais $[(M=52,05, D P=4,73,(M=$ $26,86, D P=3,06)]$. A magnitude das diferenças entre as médias nos progenitores revelou-se muito pequena na escala global e pequena na expressão do afeto $\left(\eta^{2}=0,021\right)$ e na confiança $\left(\eta^{2}=0,025\right)$.

\section{Hipótese 2: Existe uma relação positiva entre a comunicação parento-filial clara (metacomunicação) e a confiança/partilha de situações problemáticas por parte dos filhos em relação aos progenitores}

Para analisar a relação entre a clareza comunicacional (metacomunicação) e a confiança/partilha comunicacional de filhos para pais percebida pelos progenitores, utilizou-se o coeficiente de correlação de Pearson. Os pressupostos de normalidade, linearidade e homocedasticidade foram estabelecidos. Registou-se uma correlação positiva moderada entre as duas variáveis $[r(445)=0,579, p=0,001]$, com valores da subescala "confiança/partilha comunicacional de filhos para pais" a aumentarem à medida que os da subescala "metacomunicação" sobem. Obtiveram-se valores de correlação positiva moderada entre as subescalas "metacomunicação" e "confiança/partilha comunicacional de pais para filhos" $[r(445)=0,377, p=0,001]$ e entre as subescalas "confiança/partilha comunicacional de pais para filhos" e a "confiança/partilha comunicacional de filhos para pais" $[r(445)=0,391, p=0,001]$. 
Tabela 1.Teste t de Student: Diferenças de género na comunicação parento-filial (versão filial e parental)

\begin{tabular}{|c|c|c|c|c|c|c|}
\hline \multicolumn{2}{|l|}{ Sub-dimensão/Nome } & $n$ & $M$ & $D P$ & $t(\mathrm{gl})$ & $\begin{array}{l}95 \% \text { IC } \\
\text { (baixo ; alto) }\end{array}$ \\
\hline \multicolumn{7}{|l|}{ Versão: filhos em relação ao pai } \\
\hline \multirow{2}{*}{$\begin{array}{l}\text { I. Disponibilidade dos Pais para Comunicar } \\
\text { com os Filhos }\end{array}$} & Masc. & 135 & 34,69 & 4,20 & \multirow{2}{*}{$\begin{array}{l}-2,649 * \\
(309)\end{array}$} & \multirow{2}{*}{$\begin{array}{l}-2,21604 \\
-0,32663\end{array}$} \\
\hline & Femin. & 176 & 35,96 & 4,19 & & \\
\hline \multirow[t]{2}{*}{ II. Expressão do Afeto e Apoio Emocional } & Masc. & 130 & 30,72 & 5,73 & \multirow{2}{*}{$\begin{array}{l}-0,870 \\
(300)\end{array}$} & \multirow{2}{*}{$\begin{array}{l}-1,75715 \\
0,68005\end{array}$} \\
\hline & Femin. & 170 & 31,26 & 5,00 & & \\
\hline \multicolumn{7}{|l|}{ Versão: filhos em relação à mãe } \\
\hline \multirow{2}{*}{$\begin{array}{l}\text { I. Disponibilidade dos Pais para Comunicar } \\
\text { com os Filhos }\end{array}$} & Masc. & 137 & 36,14 & 4,00 & \multirow{2}{*}{$\begin{array}{l}-2,331^{*} \\
(313)\end{array}$} & \multirow{2}{*}{$\begin{array}{l}-1,85760 \\
-0,15716\end{array}$} \\
\hline & Femin. & 178 & 37,15 & 3,64 & & \\
\hline \multirow[t]{2}{*}{ II. Expressão do Afeto e Apoio Emocional } & Masc. & 134 & 33,32 & 4,87 & \multirow{2}{*}{$\begin{array}{l}-1,732 \\
(311)\end{array}$} & \multirow{2}{*}{$\begin{array}{l}-2,04735 \\
0,13048\end{array}$} \\
\hline & Femin. & 179 & 34,28 & 4,83 & & \\
\hline \multicolumn{7}{|l|}{ Versão: Progenitores } \\
\hline \multirow[t]{2}{*}{ I. Expressão do Afeto e Apoio Emocional } & Masc. & 82 & 52,05 & 4,73 & \multirow{2}{*}{$\begin{array}{l}-3,182 * \\
(450)\end{array}$} & \multirow{2}{*}{$\begin{array}{l}-2,66227 \\
-0,62936\end{array}$} \\
\hline & Femin. & 370 & 53,69 & 4,12 & & \\
\hline \multirow[t]{2}{*}{ II. Disponibilidade para a Comunicação } & Masc. & 68 & 32,80 & 3,86 & \multirow{2}{*}{$\begin{array}{l}1,097 \\
(386)\end{array}$} & \multirow{2}{*}{$\begin{array}{l}-0,43561 \\
1,53451\end{array}$} \\
\hline & Femin. & 320 & 32,26 & 3,73 & & \\
\hline \multirow[t]{2}{*}{ III. Metacomunicação } & Masc. & 84 & 33,67 & 3,34 & \multirow{2}{*}{$\begin{array}{l}-1,645 \\
(453)\end{array}$} & \multirow{2}{*}{$\begin{array}{l}-1,53591 \\
0,13609\end{array}$} \\
\hline & Femin. & 371 & 34,37 & 3,56 & & \\
\hline \multirow[t]{2}{*}{ IV. Confiança/Partilha de Pais para Filhos } & Masc. & 82 & 26,54 & 3,83 & \multirow{2}{*}{$\begin{array}{l}-1,160 \\
(448)\end{array}$} & \multirow{2}{*}{$\begin{array}{l}-1,45324 \\
0,37424\end{array}$} \\
\hline & Femin. & 368 & 27,08 & 3,80 & & \\
\hline \multirow[t]{2}{*}{ V. Confiança/Partilha de Filhos para Pais } & Masc. & 65 & 26,86 & 3,06 & \multirow{2}{*}{$\begin{array}{l}-3,505^{*} \\
(454)\end{array}$} & \multirow{2}{*}{$\begin{array}{l}-2,12635 \\
-0,59859\end{array}$} \\
\hline & Femin. & 293 & 28,23 & 3,20 & & \\
\hline
\end{tabular}

\section{Hipótese 3: As variáveis sociodemográficas "estrutura familiar", "nível socioeconômico", "local de residência" e "escolaridade" dos participantes influenciam os padrões comunicacionais entre progenitores e filhos}

Para analisar a influência das variáveis "local de residência", "nível socioeconômico", "estrutura familiar" e "escolaridade" na comunicação parento-filial recorreu-se ao teste ANOVA one-way e ao teste de Tukey para comparação múltipla das médias.

\section{Resultados na amostra das crianças}

Algumas subescalas não cumprem o pressuposto da homogeneidade, mas como a ANOVA one-way é um teste robusto, manteve-se a decisão de o aplicar, considerando a dimensão da amostra (Pallant, 2010). Os resultados obtidos na subamostra das crianças sugerem, de acordo com a Tabela 2, a existência de diferenças estatisticamente significativas em função da variável "local de residência" nas subescalas "disponibilidade dos pais para comunicar com os filhos" tendo como referência o pai $[F(2,308)=6,442, p=0,002]$ e a mãe $[F(2,312)=5,110, p=0,007] \mathrm{e}$ "expressão do afeto e apoio emocional" apenas em relação ao pai $[F(2,299)=$ $3,730, p=0,025]$. O teste de Tukey indica que as crianças que pertencem a um contexto predominantemente urbano tendem a perceber uma maior disponibilidade comunicacional por parte do pai $(M=36,87, D P=3,37)$, em comparação com as crianças que vivem em contextos medianamente urbanos $(M=35,90, D P=3,80)$ ou rurais $(M=34,43, D P=4,73)$. A disponibilidade das mães para a comunicação é percebida como sendo maior nas crianças que vivem em contextos medianamente urbanos $(M=37,35, D P=3,33)$, em contraste com o contexto predominantemente rural $(M=35,90, D P=$ $4,24)$. Por sua vez, as crianças de um contexto medianamente urbano tendem a perceber uma maior expressão dos afetos e apoio emocional dos progenitores do sexo masculino ( $M$ $=31,77, D P=5,23)$, comparativamente a crianças de contextos predominantemente rurais $(M=30,00, D P=5,51)$. $\mathrm{O}$ cálculo do tamanho do efeito, através do eta squared, revela valores que variam entre 0,02 e 0,04 , o que significa que as diferenças verificadas têm um efeito pequeno de acordo com Cohen (citado por Pallant, 2010). 
Considerando o nível socioeconômico, verificam-se algumas diferenças estatisticamente significativas nas subescalas "disponibilidade dos pais para comunicar com os filhos" tendo por referência o pai $[F(2,307)=11,850, p=0,001] \mathrm{e}$ a mãe $[F(5,404), p=0,005]$, e "expressão do afeto e apoio emocional" só em relação ao pai $[F(2,298)=4,701, p=$ $0,010]$. As crianças que pertencem à classe socioeconômica alta tendem a perceber uma maior disponibilidade para a comunicação por parte do pai $(M=36,35, D P=5,06)$ e da mãe $(M=37,78, D P=3,18)$, comparativamente às crianças de classe média [pai $(M=35,59, D P=3,90)$, mãe $(M=$ $36,75, D P=3,65)$ ] ou baixa [pai $(M=30,62, D P=5,45)$, mãe $(M=33,93, D P=6,16)]$. A expressão do afeto e apoio emocional por parte do pai parece ser percebida de uma forma significativamente menor na classe baixa $(M=27,44, D P=$ $6,47)$ comparativamente à classe média $(M=31,08, D P=$ $5,22)$ e alta $(M=32,48, D P=4,77)$. O cálculo do tamanho do efeito, através do eta squared, revela valores que variam entre 0,03 e 0,07 , o que significa que apenas o resultado relativo à "disponibilidade dos pais para comunicar com os filhos", tendo por referência o pai, tem um efeito médio; as outras diferenças têm um efeito pequeno de acordo com Cohen (citado por Pallant, 2010).

Analisando a variável "estrutura familiar" registam-se diferenças estatisticamente significativas apenas na subescala "disponibilidade dos pais para a comunicação com os filhos" relativamente ao pai $[F(3,307)=2,633, p=0,050]$. $\mathrm{O}$ teste Tukey revelou que essa variável é menos pontuada nas famílias monoparentais $(M=32,50, D P=7,14)$, seguida das famílias pós-divórcio $(M=33,39, D P=5,48)$, famílias nucleares intactas $(M=35,60, D P=3,99)$ e famílias reconstituídas $(M=35,75, D P=4,93)$. O tamanho do efeito para essa diferença é pequeno $\left(\eta^{2}=0,03\right)$.

\section{Resultados na amostra dos progenitores}

Através do teste ANOVA one-way, e considerando a variável "local de residência", encontram-se diferenças estatisticamente significativas nas subescalas "metacomunicação" $[F(2,444)=3,283, p=0,038]$ e "confiança/partilha comunicacional de pais para filhos" $[F(2,439)=3,543, p$

Tabela 2. Anova One-way: Diferenças na comunicação parento-filial ao nível das variáveis sociodemográficas dos filhos

\begin{tabular}{|c|c|c|c|c|c|}
\hline & & \multicolumn{2}{|c|}{ Versão em relação ao Pai } & \multicolumn{2}{|c|}{ Versão em relação à Mãe } \\
\hline & & I. & II. & I. & II. \\
\hline \multicolumn{6}{|c|}{ Local de Residência } \\
\hline \multirow{2}{*}{ Urbano } & $\mathrm{M}$ & 36,87 & 31,19 & 36,55 & 34,17 \\
\hline & $\mathrm{DP}$ & 3,37 & 4,59 & 4,05 & 4,55 \\
\hline \multirow{2}{*}{ Medianamente Urbano } & M & 35,90 & 31,77 & 37,35 & 34,22 \\
\hline & $\mathrm{DP}$ & 3,80 & 5,23 & 3,33 & 4,78 \\
\hline \multirow{3}{*}{ Rural } & $\mathrm{M}$ & 34,43 & 30,00 & 35,90 & 33,34 \\
\hline & DP & 4,73 & 5,51 & 4,24 & 5,02 \\
\hline & $\mathrm{F}$ & $6,442 *$ & $3,730^{*}$ & $5,110 *$ & 1,197 \\
\hline \multicolumn{6}{|c|}{ Nível Socioeconômico } \\
\hline \multirow{2}{*}{ Baixo } & M & 30,63 & 27,44 & 33,94 & 31,40 \\
\hline & $\mathrm{DP}$ & 5,45 & 6,47 & 6,16 & 6,92 \\
\hline \multirow{2}{*}{ Médio } & M & 35,59 & 31,08 & 36,75 & 33,96 \\
\hline & $\mathrm{DP}$ & 3,90 & 5,22 & 3,65 & 4,76 \\
\hline \multirow{3}{*}{ Alto } & M & 36,35 & 32,48 & 37,78 & 34,26 \\
\hline & DP & 5,06 & 4,77 & 3,18 & 4,36 \\
\hline & $\mathrm{F}$ & $11,850^{*}$ & $4,701^{*}$ & $5,404^{*}$ & 2,078 \\
\hline \multicolumn{6}{|c|}{ Estrutura Familiar } \\
\hline \multirow{2}{*}{ Nuclear Intacta } & M & 35,60 & 31,10 & 36,74 & 33,95 \\
\hline & $\mathrm{DP}$ & 3,99 & 5,13 & 3,83 & 4,72 \\
\hline \multirow{2}{*}{ Pós-Divórcio } & M & 33,39 & 29,61 & 36,77 & 34,54 \\
\hline & $\mathrm{DP}$ & 5,48 & 6,85 & 3,61 & 4,91 \\
\hline \multirow{2}{*}{ Monoparental } & M & 32,50 & 32,33 & 38,00 & 26,50 \\
\hline & $\mathrm{DP}$ & 7,14 & 9,29 & 3,46 & 16,26 \\
\hline \multirow{3}{*}{ Reconstituída } & M & 35,75 & 31,75 & 35,82 & 32,61 \\
\hline & DP & 4,93 & 5,52 & 4,30 & 5,07 \\
\hline & $\mathrm{F}$ & $2,633^{*}$ & 0,715 & 0,424 & 2,134 \\
\hline
\end{tabular}


$=0,030]$, tal como se pode ver na Tabela 3. Os progenitores que pertencem a um contexto predominantemente urbano tendem a perceber uma maior clareza comunicacional (metacomunicação) com os seus filhos $(M=34,66, D P=3,26)$ do que aqueles que vivem em contextos medianamente urbanos $(M=33,91, D P=3,88)$ ou predominantemente rurais $(M=33,56, D P=3,25)$. Por outro lado, a "confiança/ partilha comunicacional de pais para filhos" parece ser menor em contextos predominantemente urbanos $(M=26,54, D P$ $=3,65)$ em comparação com os contextos medianamente urbanos $(M=27,56, D P=3,17)$. Apesar de as diferenças serem estatisticamente significativas, o tamanho do efeito é pequeno para ambas $\left(\eta^{2}=0,01 ; \eta^{2}=0,02\right)$.

$\mathrm{Na}$ análise da comunicação parento-filial em função do nível socioeconômico verificam-se diferenças estatisticamente significativas apenas na subescala "confiança/partilha comunicacional de pais para filhos" $[F(2,442)=8,306, p=$ $0,001]$, com os progenitores que pertencem à classe socioeconômica baixa a demonstrarem mais confiança nos filhos $(M=28,39, D P=3,89)$ do que os progenitores da classe socioeconômica média $(M=26,72, D P=3,72)$ ou alta $(M=$ $25,74, D P=3,29)$. O tamanho do efeito para esses resultados é pequeno $\left(\eta^{2}=0,01\right)$.

Por fim, ao nível da escolaridade dos progenitores registam-se diferenças estatisticamente significativas nas subescalas "metacomunicação" $[F(7,440)=6,972, p=$ 0,001], "confiança/partilha comunicacional de pais para filhos" $[F(7,435)=5,315, p=0,001]$ e "confiança/partilha comunicacional de filhos para pais" $[F(7,441)=2,605, p$ $=0,012]$. Os resultados no teste de Tukey indicam que os progenitores que completaram quatro anos de ensino básico tendem a estabelecer uma comunicação significativamente menos clara com os filhos $(M=29,43, D P=6,15)$ em comparação com os progenitores que estudaram durante seis anos $(M=34,17, D P=2,44)$, nove anos $(M=33,66, D P=4,17)$, 12 anos $(M=34,52, D P=3,24)$, e também em comparação com os progenitores que completaram a licenciatura $(M=$ $34,53, D P=2,96)$, o mestrado $(M=34,61, D P=3,33)$ e o doutoramento $(M=34,52, D P=2,98)$. Ao nível da "confiança/partilha parental", os progenitores que completaram nove anos de ensino básico destacam-se por revelarem maior confiança e partilha de assuntos problemáticos com os filhos $(M=28,75, D P=3,69)$, comparativamente aos progenitores que completaram 12 anos de estudos $(M=27,99, D P=$ $3,23)$, a licenciatura $(M=26,13, D P=3,88)$ e o mestrado $(M=25,60, D P=3,62)$. Relativamente à "confiança/partilha dos pais para os filhos" constata-se que aqueles que completaram apenas os quatro anos de ensino básico se destacam por terem uma baixa percepção nessa subescala ( $M$ $=25,45, D P=4,78)$ comparativamente aos que concluíram seis anos $(M=28,48, D P=3,41)$, nove anos $(M=28,30$, $D P=3,20), 12$ anos de estudos básicos $(M=28,20, D P=$ $3,29)$, a licenciatura $(M=28,91, D P=2,93)$ e o mestrado $(M=28,95, D P=2,54)$. Nesse caso, o cálculo do tamanho do efeito revela que as subescalas "metacomunicação" $\left(\eta^{2}\right.$ $=0,10)$ e "confiança/partilha comunicacional de pais para filhos" $\left(\eta^{2}=0,08\right)$ têm um efeito médio enquanto a subescala "confiança/partilha comunicacional de filhos para pais" tem um efeito pequeno $\left(\eta^{2}=0,04\right)$. A variável "estrutura familiar" não revelou qualquer resultado estatisticamente significativo na versão parental do COMPA.

\section{Discussão}

O principal objetivo do presente estudo foi identificar e comparar as percepções que progenitores e crianças (entre os 7 e os 11 anos) têm sobre a comunicação parento-filial, através do teste de três hipóteses de investigação. Para tal, recorreu-se à Escala de Avaliação da Comunicação na $\mathrm{Pa}-$ rentalidade (COMPA-P e COMPA-C).

A literatura indica que, apesar do progressivo envolvimento da figura paterna nas atividades e interações com os filhos, as mães tendem a manter um papel de destaque na comunicação parento-filial (McNaught, 2000; Patrick et al., 2005). Essas diferenças em função do sexo registaram-se nas crianças da amostra em estudo, com as meninas a obterem valores mais elevados na escala COMPA comparativamente aos meninos, emergindo a preferência da comunicação com as mães. Esses resultados podem advir das atribuições sociais aos papéis parentais uma vez que, nas sociedades ocidentais e, particularmente em Portugal, há ainda uma diferenciação nos papéis e funções de pai e mãe. Verifica-se, por exemplo, que as mulheres são mais incentivadas a partilhar as suas emoções e sentimentos, comparativamente aos homens (McNaughton, 2000). Socialmente, é esperado que as mulheres sejam mais atentas e respondam às necessidades básicas das crianças (e.g. cuidados de saúde, higiene), enquanto os homens/pais tendem a gerir as dimensões que envolvem poder (e.g. estabelecimento de regras e limites, monitorização dos resultados académicos) (McNaughton, 2000). Essas atribuições e representações sociais em torno dos papéis de gênero influenciam o exercício da parentalidade e a forma como pais/mães e filhos se relacionam numa dada cultura. Assim, as mães participantes neste estudo destacam-se relativamente aos pais por se considerarem mais atentas às necessidades básicas e quotidianas dos filhos, estabelecendo uma comunicação mais positiva e de maior partilha, comparativamente aos pais. $\mathrm{O}$ mesmo se aplica às meninas, que percebem uma maior disponibilidade parental para a comunicação, tanto por parte das mães como por parte dos pais. De acordo com McNaughton (2000), esse resultado poderá estar relacionado com o fato de as meninas partilharem mais assuntos com os progenitores do que os meninos, logo, percebem uma maior disponibilidade parental do que as crianças do sexo masculino.

A literatura (Bumpus \& Hill, 2008; Watzlawick et al., $1967 / 1993$ ) indica que existe uma relação positiva entre a confiança/partilha de situações problemáticas por parte dos filhos e uma comunicação parento-filial caracterizada por um registo claro, sem distorções comunicacionais, isto é, uma comunicação que clarifica não só os conteúdos como também a relação, evitando mal-entendidos entre os membros envolvidos na interação comunicacional. Os progenitores que indicam uma comunicação clara com os filhos, ou seja, que referem metacomunicar tendem a perceber maior confiança por parte destes. A metacomunicação é favorável ao bem-estar e desenvolvimento individual e familiar, 
Tabela 3. Anova One-way: Diferenças na comunicação parento-filial ao nível das variáveis sociodemográficas dos progenitores

\begin{tabular}{|c|c|c|c|c|c|c|}
\hline & & I. & II. & III. & IV. & V. \\
\hline \multicolumn{7}{|c|}{ Local de Residência } \\
\hline \multirow{2}{*}{ Urbano } & $M$ & 53,71 & 32,08 & 34,66 & 26,54 & 28,10 \\
\hline & $D P$ & 3,92 & 3,59 & 3,26 & 3,65 & 31,93 \\
\hline \multirow{2}{*}{ Medianamente Urbano } & $M$ & 53,19 & 32,10 & 33,91 & 27,56 & 28,07 \\
\hline & $D P$ & 4,70 & 4,06 & 3,88 & 4,09 & 3,28 \\
\hline \multirow{3}{*}{ Rural } & $M$ & 52,69 & 31,77 & 33,56 & 26,88 & 27,38 \\
\hline & $D P$ & 4,29 & 3,11 & 3,25 & 3,17 & 3,22 \\
\hline & $F$ & 1,439 & 2,412 & $3,283^{*}$ & $3,543^{*}$ & 1,082 \\
\hline \multicolumn{7}{|c|}{ Nível Socioeconômico } \\
\hline \multirow{2}{*}{ Baixo } & $M$ & 53,27 & 32,83 & 33,61 & 28,39 & 28,11 \\
\hline & $D P$ & 5,42 & 4,29 & 4,92 & 3,89 & 3,98 \\
\hline \multirow{2}{*}{ Médio } & $M$ & 53,44 & 32,18 & 34,44 & 26,72 & 27,91 \\
\hline & $D P$ & 3,96 & 3,64 & 3,10 & 3,72 & 3,05 \\
\hline \multirow{3}{*}{ Alto } & $M$ & 53,68 & 33,59 & 33,96 & 25,74 & 28,68 \\
\hline & $D P$ & 4,25 & 3,20 & 3,21 & 3,29 & 2,48 \\
\hline & $F$ & 0,105 & 2,279 & 1,975 & $8,306^{*}$ & 0,791 \\
\hline \multicolumn{7}{|c|}{ Estrutura Familiar } \\
\hline \multirow{2}{*}{ Nuclear Intacta } & $M$ & 53,36 & 32,50 & 34,13 & 26,94 & 27,90 \\
\hline & $D P$ & 4,27 & 3,66 & 3,54 & 3,75 & 3,19 \\
\hline \multirow{2}{*}{ Pós-Divórcio } & $M$ & 53,49 & 31,45 & 35,33 & 26,86 & 28,74 \\
\hline & $D P$ & 4,41 & 4,18 & 2,98 & 4,42 & 2,97 \\
\hline \multirow{2}{*}{ Monoparental } & $M$ & 54,20 & 32,71 & 34,00 & 30,20 & 28,33 \\
\hline & $D P$ & 5,97 & 3,73 & 4,24 & 3,19 & 4,03 \\
\hline \multirow{3}{*}{ Reconstituída } & $M$ & 54,00 & 31,13 & 33,56 & 27.44 & 27,67 \\
\hline & $D P$ & 3,67 & 5,00 & 4,67 & 3,36 & 4,56 \\
\hline & $F$ & 0,134 & 1,316 & 1,713 & 1.267 & 1,031 \\
\hline \multicolumn{7}{|c|}{ Escolaridade } \\
\hline \multirow{2}{*}{4 anos } & $M$ & 51,10 & 31,50 & 29,43 & 26,42 & 25,45 \\
\hline & $D P$ & 7,67 & 5,26 & 6,15 & 5,05 & 4,78 \\
\hline \multirow{2}{*}{6 anos } & $M$ & 52,95 & 33,15 & 34,17 & 28,00 & 28,48 \\
\hline & $D P$ & 4,73 & 3,80 & 2,44 & 3,68 & 3,41 \\
\hline \multirow{2}{*}{9 anos } & $M$ & 53.25 & 33,82 & 33,66 & 28,75 & 28,30 \\
\hline & $D P$ & 4,61 & 3,73 & 4,17 & 3,69 & 3,20 \\
\hline \multirow{2}{*}{12 anos } & $M$ & 53,52 & 32,49 & 34,52 & 27,99 & 28,19 \\
\hline & $D P$ & 4,17 & 3,97 & 3,24 & 3,23 & 3,29 \\
\hline \multirow{2}{*}{ Licenciatura } & $M$ & 53,52 & 31,94 & 34,53 & 26,13 & 27,91 \\
\hline & $D P$ & 3,67 & 3,54 & 2,96 & 3,88 & 2,93 \\
\hline \multirow{2}{*}{ Mestrado } & $M$ & 52,92 & 32,83 & 34,61 & 25,60 & 28,95 \\
\hline & $D P$ & 4,21 & 3,13 & 3,33 & 3,62 & 2,54 \\
\hline \multirow{3}{*}{ Doutoramento } & $M$ & 53,52 & 31,62 & 34,52 & 26,00 & 28,00 \\
\hline & $D P$ & 3,43 & 3,17 & 2,98 & 3,65 & 2,76 \\
\hline & $F$ & 1,071 & 1,542 & $6,972 *$ & $5,315^{*}$ & $2,605^{*}$ \\
\hline
\end{tabular}

particularmente nessa etapa do ciclo vital de famílias com filhos na escola, caracterizada pela abertura do sistema familiar ao exterior (Bumpus \& Hill, 2008; Patrick et al., 2005; Watzlawick et al., 1967/1993). A metacomunicação parece facilitar a confiança e a abertura na comunicação verbal e não-verbal entre progenitores e filhos, facilitando uma monitorização parental mais eficaz sobre o quotidiano das crianças. Esses padrões comunicacionais são apontados como fatores de prevenção de comportamentos sociais e familiares desajustados (Bumpus \& Hill, 2008). A investigação desenvolvida por Liau, Khoo e Ang (2008) indica que as novas tecnologias podem ser fatores desafiantes para as estratégias parentais educativas e respectiva monitorização. Apesar da metacomunicação poder estar relacionada com a percepção parental de maior confiança/partilha por parte dos filhos, é importante ponderar que o acesso precoce às novas tecnologias (e.g. internet) dificulta a supervisão e o controlo dos progenitores. Dessa forma, é importante que a metacomunicação no contexto familiar acompanhe a progressiva disseminação dessas vias de comunicação. $\mathrm{O}$ estudo realizado por Liau et al. (2008) realça a necessidade de reconceptualizar a monitorização parental e melhorar a comunicação parento-filial perante esse novo "membro" da família, que é a internet. 
Um outro dado de interesse no estudo desenvolvido, aponta para a influência das variáveis sociodemográficas, tais como local de residência, nível socioeconômico, estrutura familiar e escolaridade dos progenitores na comunicação parento-filial. Um dos resultados em destaque é a percepção que as crianças oriundas de áreas urbanas têm sobre a comunicação estabelecida com as figuras parentais. Verifica-se que essas crianças percebem de uma forma mais positiva a comunicação estabelecida com os respectivos progenitores (ao nível da disponibilidade parental para a comunicação e da expressão do afeto e apoio emocional), comparativamente às crianças de áreas rurais. Esse dado é coerente com o estudo de Wamoyi et al. (2010), no qual se verificou que as crianças e progenitores de contextos rurais têm mais dificuldade em abordar determinados temas, nomeadamente tópicos relacionados com a sexualidade, do que as crianças e progenitores de contextos urbanos. Os contextos rurais são caracterizados por um menor acesso à informação e à globalização pois estão, frequentemente, afastados dos polos onde se centraliza o conhecimento e a inovação (contextos urbanos). Este aspecto pode justificar a menor disponibilidade parental para a comunicação e a menor expressão de afeto e apoio emocional. Além disto, Whitbeck, Simons, Conger, Wickrama e Elder (1997) referem que essa diferença poderá estar relacionada com aspectos como o trabalho e as dificuldades econômicas que, também, caracterizam os contextos rurais, uma vez que o estatuto socioeconômico é apontado como uma variável igualmente importante na gestão da comunicação parento-filial (Magnuson \& Duncan, 2002). De fato, no presente estudo verifica-se que as famílias provenientes do nível socioeconômico baixo tendem a manifestar uma comunicação global menos satisfatória, comparativamente às famílias de nível socioeconômico médio e/ou alto.

Contudo, os resultados obtidos na amostra dos progenitores revelam um dado curioso: os progenitores que pertencem ao estatuto socioeconômico baixo referem partilhar mais os seus problemas com os filhos do que aqueles que provêm de um estatuto socioeconômico médio e/ou alto. Essa diferença pode ser justificada pelo fato de as famílias de nível socioeconômico baixo terem uma rede social de apoio pobre, o que faz com que os adultos vejam nos seus filhos, ainda que crianças, as pessoas acessíveis com quem podem partilhar as suas preocupações, alegrias, experiências (Brown \& Lynn, 2010). Porém, e ainda que de forma inconsciente, essa interação parento-filial pode dar origem a processos de parentificação das crianças, que são desfavoráveis ao seu bem-estar e desenvolvimento adequado (Peris, Cummings, Goeke-Morey, \& Emery, 2008).

Um outro resultado interessante surge relativamente à influência da "escolaridade" das figuras parentais sobre as interações comunicacionais. Verificou-se que os progenitores que concluíram apenas os quatro primeiros anos de ensino tendem a metacomunicar menos com os seus filhos do que os restantes progenitores, tal como é sugerido por Jiménez e Delgado (2002). Quanto mais baixa é a escolaridade dos pais, maiores serão as suas dificuldades na organização de um pensamento complexo e na estruturação de uma comunicação clara (Dekovic \& Gerris, 1992). Esse resultado reforça a existência de algumas dificuldades comunicacionais entre pais e filhos em contextos menos favorecidos, nomeadamente ao nível socioeconômico, residencial e educativo. Os resultados obtidos na escala COMPA nas duas amostras (progenitores e crianças) sugerem que as famílias que vivem em contextos rurais, que pertencem ao nível socioeconómico baixo e cujos progenitores apenas completaram quatro anos de escolaridade tendem a estabelecer uma comunicação parento-filial global mais enfraquecida, embora existam algumas variações ao nível das subdimensões da escala COMPA (apesar dos progenitores de nível socioeconômico baixo pontuarem menos na comunicação global, destacam-se por revelar uma pontuação elevada na dimensão "confiança/partilha de pais para filhos").

A variável "estrutura familiar" influencia também a comunicação parento-filial, com os resultados a mostrarem que as crianças que vivem em famílias monoparentais pontuam significativamente menos na subescala "expressão do afeto e apoio emocional" tendo por referência o pai, comparativamente às crianças de famílias pós-divórcio. Esse resultado é compreensível atendendo ao fato de que a amostra de famílias monoparentais estudada é maioritariamente composta por mães. Por esse motivo, as crianças podem perceber uma menor clareza comunicacional com os progenitores do sexo masculino por estes serem uma figura ausente do agregado familiar. Por outro lado, e ao contrário do que é sugerido pela literatura empírica (Afifi et al., 2006; Dunn et al., 2001; Eldar-Avidan et al., 2009; McManus \& Nussbaum, 2011), não se registaram diferenças significativas nos resultados da escala COMPA em função das diferentes estruturas familiares, na amostra dos progenitores. Alarcão (2006) sugere que a qualidade da comunicação parento-filial na etapa do ciclo vital "família com filhos na escola" pode variar em função do tipo de família uma vez que as diferentes configurações familiares têm "algumas particularidades que ultrapassam as singularidades de cada família" (p. 204). Nair e Murray (2005), analisando o impacto do divórcio na qualidade da vinculação entre mães e filhos, constataram que em famílias divorciadas as mães tendem a manifestar níveis elevados de estresse e depressão e utilizam significativamente menos estratégias positivas no exercício da parentalidade do que as mães que pertencem a núcleos familiares intactos. As crianças de famílias divorciadas apresentavam valores mais baixos ao nível da vinculação segura com as mães. Esses resultados sustentam a ideia de que famílias com uma estrutura diferente da tradicional "nuclear intacta" tendem a enfrentar mais desafios e riscos (Nair \& Murray, 2005). Porém esses indicadores não se refletem inteiramente no presente estudo. Poderão ser avançadas várias hipóteses para esse resultado, a começar pela mudança de perspectiva no atual panorama social relativa ao conceito de família. De acordo com Torres (2010), a sociedade contemporânea marcou um ciclo de mudanças no que diz respeito à forma como vê o casamento e a família. Torres $(2010$, p. 25$)$ refere verificar-se, por toda a Europa, uma "maior valorização dos interesses individuais (...), exigência de posições simétricas entre homens e mulheres e desvalorização ou resistência a formas externas de imposição e condicionamento", perspectiva que coloca em causa o conceito de família tradicional e a própria finalidade do casamento. Essa mudança de paradigma social reconhece as diferentes estruturas familiares condições e competências que, até agora, eram negadas ou ignoradas. A perspectiva centrada nas competências, e cada vez menos no défice, permite 
às novas constelações familiares adaptarem-se à sua estrutura de uma forma tendencialmente positiva e menos estressante.

A análise dos resultados referidos deve ser feita tendo em consideração algumas limitações. Uma delas prende-se com a constituição da amostra já que, para além de ter sido uma amostra por conveniência, o número de participantes em cada condição das variáveis sociodemográficas analisadas limita a representatividade de cada uma das categorias que as compõem. Além disso, o estudo peca por alguns desequilíbrios ao nível da variável sexo, nomeadamente, no número de mães e de pais participantes. $\mathrm{O}$ fato de participarem mais mães do que pais pode influenciar os resultados. A maior disponibilidade por parte das figuras maternas para colaborar na presente investigação não deixa de ser um dado interessante, pois o crescente envolvimento paterno nas atividades dos filhos é sustentado pela literatura e mesmo pela prática clínica. Por outro lado, verifica-se uma grande adesão de participantes do sexo masculino que pertencem a famílias pós-divórcio, ou seja, que exercem a parentalidade de uma forma mais autônoma/individual pois não integram um par parental. Por fim, importa realçar uma outra limitação do estudo, nomeadamente o facto de se utilizarem subamostras independentes de pais e de filhos, isto é, sem grau de parentesco entre si. Esse aspecto impossibilita a comparação das dimensões da comunicação ao nível da coparentalidade, assim como a análise diádica (pai/mãe - filhos) e triádica (pai - mãe - filhos) das percepções de progenitores e de filhos sobre a comunicação que mantêm.

\section{Conclusões}

A presente investigação permitiu a análise da comunicação parento-filial em famílias com filhos em idade escolar. Os resultados obtidos são úteis para os profissionais que trabalham direta ou indiretamente com essas famílias, como por exemplo, terapeutas familiares, assistentes de segurança social, professores, entre outros. Alguns dos resultados implicam uma atenção reforçada às relações familiares de indivíduos desfavorecidos em termos sociais, econômicos e educativos. Os indicadores do presente estudo funcionam ainda como sinal de alerta para os clínicos, permitindo a identificação das necessidades comunicacionais tanto dos progenitores como dos filhos.

A investigação futura deve promover o estudo desse tema com base em amostras de maior dimensão e equidade na distribuição por sexo dos respondentes, abarcando população geral e amostras específicas do contexto clínico/forense e que integrem todos os elementos do mesmo agregado familiar para obter, assim, um registo mais claro da comunicação parento-filial. É pertinente promover estudos que cruzem a variável "comunicação parento-filial”" com outras dimensões centrais no exercício da parentalidade, nomeadamente a vinculação e as práticas educativas parentais no sentido de promover o conhecimento científico aprofundado de uma das dimensões mais relevantes para o funcionamento familiar. Além disto, poderá ser interessante analisar a relação entre as subdimensões da escala COMPA com as competências sociais de crianças em idade escolar.

\section{Referências}

Afifi, T. D., Huber, F. N., \& Ohs, J. (2006). Parents' and adolescents' communication with each other about divorce-related stressors and its impact on their ability to cope positively with the divorce. Journal of Divorce and Remarriage, 45(1/2), 1-30.

Alarcão, M. (2006). (Des) equilíbrios Familiares (3 ${ }^{\mathrm{a}}$ ed.). Coimbra: Quarteto.

Brown, E. D., \& Lynn, T. K. (2010). Daily poverty-related stress and mood for low-income parents, as a function of the presence of a cohabiting partner relationship. Individual Differences Research, 8(4), 204-213.

Bumpus, M. F., \& Hill, L. G. (2008). Secrecy and parent-child communication during middle childhood: associations with parental knowledge and child adjustment. Parenting: Science and Practice, 8, 93-116.

Camacho, I., \& Matos, M. G. (2007). Práticas parentais educativas, fobia social e rendimento académico em adolescentes. Revista Brasileira de Terapias Cognitivas, 3(3), 1-15.

Carr, A. (2006). Family Therapy. Concepts, Process and Practice. $\left(2^{\text {nd }}\right.$ Ed. $)$. Chichester, England: John Wiley \& Sons.

Dekovic, M., \& Gerris, J. R. M. (1992). Parental reasoning complexity, social class, and child-rearing behaviors. Journal of Marriage and the Family, 54, 675-685.

Dunn, J., Davies, L. C., O’Connor, T. G., \& Sturgess, W. (2001). Family lives and friendships: the perspectives of children in step-, single-parent, and nonstep families. Journal of Family Psychology, 15(2), 272-287.

Eldar-Avidan, D., Haj-Yahia, M. M., \& Greenbaum, C. W. (2009). Divorce is a part of my life... Resilience, survival, and vulnerability: young adults' perception of the implications of parental divorce. Journal of Marital and Family Therapy, 35(1), 30-46.

Fiske, J. (2005). Introdução ao Estudo da Comunicação. (9a Ed.). Lisboa: ASA Editores. (Trabalho original publicado em 1993)

Gentzler, A. L., Contreras-Grau, J. M., Kerns, K. A., \& Weimer, B. L. (2005). Parent-child emotional communication and children's coping in middle childhood. Social Development, 14(4), 591-612.

Herbert, M. (2004). Parenting Across the Lifespan. In M. Hoghughi, \& N. Long (Eds.), Handbook of Parenting. Theory and Research for Practice (pp. 55-72). London: Sage Publications.

Hillaker, B. D., Brophy-Herb, H. E., Villarruel, F. A., \& Haas, B. E. (2008). The contributions of parenting to social competencies and positive values in middle school youth: positive family communication, maintaining standards, and supportive family relationships. Family Relations, 57(5), 591-601. doi:10.1111/ j.1741-3729.2008.00525.x

Jiménez, A. P., \& Delgado, A. O. (2002). Comunicación y conflicto familiar durante la adolescencia. Anales de Psicologia, 18(2), 215-231.

Kerr, M., Stattin, H., \& Özdemir, M. (2012). Perceived parenting style and adolescent adjustment: Revisiting directions of effects and the role of parental knowledge. Developmental Psychology, 48(6), 1540-1553. doi:10.1037/a0027720

Kirkman, M., Rosenthal, D. A., \& Feldman, S. (2005). Being open with your mouth shut: The meaning of 'openness' in family communication about sexuality. Sex Education, 5(1), 49-66. doi:10.1080/1468181042000301885 
Liau, A., Khoo, A., \& Ang, P. (2008). Parental awareness and monitoring of adolescent internet use. Current Psychology, 27(4), 217-233.

Magnuson, K. A., \& Duncan, G. J. (2002). Parents in Poverty. In M. Bornstein (Ed.), Handbook of Parenting - Volume IIII. Social Conditions and Applied Parenting (2 ${ }^{\text {nd }}$ Ed. pp. 95-122). London: Lawrence.

McManus, T. G., \& Nussbaum, J. F. (2011). Social support expectations and strategic ambiguity in parent-young adult child divorce-related stressor conversations. Journal of Divorce and Remarriage, 52(4), 244-270.

McNaughton, J. (2000). Gender differences in parent child communication patterns. Journal of Undergraduate Research, 3, 25-32.

Nair, H., \& Murray, A. D. (2005). Predictors of attachment security in preschool children from intact and divorced families. Journal of Genetic Psychology, 166(3), 245-263.

Pais-Ribeiro, J. (2007). Metodologia de investigação em psicologia e saúde. Porto: Legis Editora.

Pallant, J. (2010). SPSS Survival Manual. A step by step guide to data analysis using SPSS for program (Version 18). (4 ${ }^{\text {th }} \mathrm{Ed}$.). Philadelphia: Open University Press.

Patrick, M. R., Snyder, J., Schrepferman, L. M., \& Snyder, J. (2005). The joint contribution of early parental warmth, communication and tracking, and early child conduct problems on monitoring in late childhood. Child Development, 76(5), 999-1014.

Peris, T. S., Cummings, E. M., Goeke-Morey, M. C., \& Emery, R. E. (2008). Marital conflict and support seeking by parents in adolescence: empirical support for the parentification construct. Journal of Family Psychology, 22(3), 633-642.

Portugal A., \& Alberto I. (2013). Comunicação entre pais e filhos adolescentes: um estudo quantitativo de uma amostra Portuguesa. Manuscript submitted for publication.

Relvas, A. P. (1996). O Ciclo Vital da Família. Perspectiva Sistémica. Porto: Afrontamento.

Riesch, S. K., Anderson, L. S., \& Krueger, H. A. (2006). Parentchild communication processes: preventing children's health-risk behavior. Journal for Specialists in Pediatric Nursing, 11(1), 41-56.

Segrin, C., \& Flora, J. (2005). Family Communication. London: Lawrence Erlbaum Associates.

Stafford, L. (2004). Communication competencies and sociocultural priorities of middle childhood. In A. Vangelisti (Ed.), Handbook of Family Communication. (pp. 311-332). London: Lawrence Erlbaum Associates.
Stamp, G. (2004). Theories of family relationships and a family relationships theoretical model. In A. Vangelisti (Ed.), Handbook of Family Communication. (pp. 1-30). London: Lawrence Erlbaum Associates.

Streeck, J. (2002). Culture, meaning, and interpersonal communication. In M. L. Knapp, \& J. A. Daly (Eds.), The handbook of interpersonal communication ( $3^{\text {rd }}$ Ed., pp. 300-336). Thousand Oaks, CA: Sage.

Taborda Simões, M. C. T., Martins, R. C., \& Formosinho, M. D. (2006). Regulação do exercício do poder paternal: aspectos jurídicos e avaliação psicológica. In A. C. Fonseca, M. R. Simões, M. C. Taborda Simões, \& M. S. Pinho (Eds.), Psicología Forense (pp. 497-518). Coimbra: Almedina.

Torres, A. M. C. (2010). Mudanças na Família. O Privado na Agenda Pública. Provas de agregação em Sociologia apresentadas ao Departamento de Sociologia do Instituto Universitário de Lisboa.

Wamoyi, J., Fenwick, A., Urassa, M., Zaba, B., \& Stones, W. (2010). Parent-child communication about sexual and reproductive health in rural Tanzania:iImplications for young people's sexual health interventions. Reproductive Health, 7(6), 1-18. doi:10.1186/1742-4755-7-6

Watzlawick, P., Beavin, J. B., \& Jackson, D. (1993). Pragmatics of Human Communication: A study of international patterns, pathologies, and paradoxes. New York: W. W. Norton \& Company. (Trabalho original publicado em 1967)

Whitbeck, L. B., Simons, R. L., Conger, R. D., Wickrama, K., Ackley, K. A., \& Elder, G. H. (1997). The effects of parents' working conditions and family economic hardship on parenting behaviors and children's self-efficacy. Social Psychology Quarterly, 60(4), 291-303. 